\title{
Approach to Context Computation for Implicit Context-sensitive Graph Grammars
}

\author{
Yang Zou, Xiaoqin Zeng, Yufeng Liu, and Huiyi Liu \\ Institute of Intelligence Science and Technology \\ Hohai University \\ Nanjing, P. R. China
}

\begin{abstract}
Context-sensitive graph grammars are appropriate formalisms for specifying visual programming languages, as they are intuitive, rigorous, and expressive. Nevertheless, some of the formalisms whose contexts are implicitly or incompletely represented in productions, called implicit context-sensitive graph grammars, suffer inherent weakness in intuitiveness or limitations in parsing efficiency. Context tends to be a conceivable way to address this issue. Based on the formalization of context, this paper proposes an approach to the computation of context for implicit context-sensitive graph grammars. Moreover, the complexity of the four algorithms comprising the approach is analyzed and the applicability of the approach is discussed. The proposed approach paves the way for the applications of context in implicit graph grammar formalisms, such as facilitating comprehension of graph grammars and improving efficiency of general parsing algorithms.
\end{abstract}

Keywords - visual languages; implicit context-sensitive graph grammars; context computation; algorithm; complexity

\section{INTRODUCTION}

Visual Programming Languages (VPLs), which usually handle objects that do not possess inherent visual representation [1], have been frequently utilized in many fields of computer science including software engineering. Various approaches have been proposed to formally specify and parsing VPLs [2-4]. As a natural extension of formal grammar theory, graph grammars offer the mechanisms for formal specification and parsing of VPLs [5], just like formal grammars do for string languages. However, the extension from one-dimensional string-based formal grammars to two-dimensional graph grammars brings about a few novel challenges, especially the embedding problem. Quite a few graph grammar formalisms have been proposed in the literature [5-9]. From the perspective of usability, there is still room for these formalisms to be ameliorated in expressive power or computing efficiency.

Most existing graph grammar formalisms are context-free or context-sensitive. The expressive power of a graph grammar lies on the type it belongs to as well as the embedding mechanism it chooses [10-11]. Among all the categories of embedding mechanisms that vary in complexity and power, invariant embedding is the least complex one and most commonly employed in graph grammar formalisms. Contextsensitive graph grammars tend to be more expressive than context-free ones, when confined to identical less complex embedding mechanisms and invariant embedding in particular. As context-free graph grammars have difficulty in specifying a

\footnotetext{
DOI reference number: 10.18293/DMSVIVA2019-017
}

large portion of graphical VPLs [8-9], recent research in this field focus more on context-sensitive graph grammars [12-15].

A graph grammar consists of a set of productions, each of which is a pair of graphs, called left graph and right graph, together with an embedding expression. In context-sensitive graph grammars, the contexts pertaining to a production generally refer to the neighboring subgraphs of the rewritten portion of its left graph in potential host graphs [16], which describe the situations under which the production can be applied. A host graph is a graph that is being rewritten by some graph grammar in the process of derivation or parsing. However, the context portion of a production, i.e., the remainder of the left graph minus the rewritten portion, is commonly not a direct copy of the contexts for the sake of conciseness of productions and easiness of embedding.

The most representative context-sensitive graph grammar formalisms are Layered Graph Grammar (LGG) [8] and Reserved Graph Grammar (RGG) [9]. To solve the embedding problem, LGG identically involves in the left and right graphs of a production its immediate context and imposing a dangling edge condition on redex definition, which guarantees that dangling edges never occur in rewritten host graphs. Generally, a redex is a subgraph in a host graph that is isomorphic to the left or right graph of a production. RGG is commonly viewed as an improvement over LGG in respect of succinctness of specification and efficiency of parsing algorithm. Rather than directly involving contexts in productions just as LGG does, RGG formalism invents a particular two-level node structure coupled with a marking technique to indirectly specify the context of a production by identically distributing a set of marked vertices into the left and right graphs. The vertices establish a one-to-one correspondence between the two graphs in terms of their marks. Thus, the embedding problem is solved through this mechanism together with a dedicated embedding rule. Other context-sensitive formalisms include Edge-based Graph Grammar (EGG) [17-18], Context-Attributed Graph grammar (CAGG) [19], Contextual Layered Graph Grammar (CLGG) [20], and Spatial Graph Grammars (SGG) [12]. To tackle the embedding problem, EGG identically augment a set of marked dangling edges to both the left and right graphs of a production, whereas CAGG introduces attributes of nodes to establish a correspondence between the two graphs of a production. CLGG and SGG are extensions of LGG and RGG, respectively. Based on LGG, CLGG supports three extra 
mechanisms, which can be employed to define more complex VPLs. SGG extends RGG by augmenting its productions with a spatial specification mechanism, with which it can explicitly describe both structural and spatial relationships for VPLs.

In terms of how the context portion of a production is dealt with, the preceding formalisms can be classified into two categories: explicit and implicit [21]. The former are those that directly enclose the complete immediate contexts as its context portion in a production, whereas the latter refers to the ones in which the context portion is expressed as specifically tailored (i.e., incomplete) immediate contexts, newly introduced notations or attributes adhered to the rewritten portion. Readily, LGG and RGG are the typical examples of the former and the latter, respectively.

An inherent weakness of implicit formalisms is that they are not intuitive, which arises from the fact that the context portion of a production is not the complete immediate contexts. In RGG, the context portion is a set of marked vertices. Vertices are explained to be connecting points of edges, but their exact meaning is left undefined. Therefore, the selection, arrangement and marking of vertices within a node become a challenge in the design of productions. Similar situations arise in other implicit formalisms. Moreover, as actual immediate contexts are absent in productions, it is rather difficult for users to exactly comprehend the language of a given graph grammar.

The exploitation of context is a conceivable way to address the above issues. Obviously, context is useful in several situations. Context can be used to make up the deficiency in intuitiveness so as to facilitate the comprehension and design of implicit graph grammars. Furthermore, context can be utilized to reduce backtracking in general parsing algorithms by context matching, thus improving the parsing efficiency. In the literature [21], a formal definition of context is proposed and the properties are characterized, which provide a solid theoretical foundation for the computation of context. Nevertheless, due to the complexity of context formalization, an explicit method for the computation of context is apparently a necessity for the purpose of practical usage.

To this end, on the basis of RGG formalism, an approach for the computation of context for implicit graph grammars is proposed in this paper. This is a subsequent research on context, and the technical contributions are as follows: It proposes a concrete approach for context computation, which consists of four partially ordered algorithms with each one being dependent on its predecessors. Moreover, it provides the time complexities of the algorithms and discusses the applicability of the approach. Besides, this method can be generalized to other implicit graph grammar formalisms. Therefore, it paves the way for the application of context in implicit graph grammar formalisms.

The remainder of the paper is organized as follows: Section 2 reviews the RGG formalism and excerpts the formal definition of context. Section 3 proposes an approach that consists of four algorithms to the computation of context. Section 4 addresses the complexities of the algorithms. Section 5 discusses the applicability of the algorithms. Finally, section 6 concludes the paper and proposes future research.

\section{PRELIMINARIES}

\section{A. The RGG Formalism}

A graph grammar consists of an initial graph and a collection of productions (graph rewriting rules). Each production has two graphs called left graph and right graph respectively, and can be applied to another graph called host graph. Every node in a production is either a terminal or a non-terminal node. A graph grammar defines a graph language composed of those graphs that can be derived from the initial graph by repeated applications of the productions and whose nodes are all terminal ones. A redex is a subgraph in a host graph that is isomorphic to the left or right graph of a production.

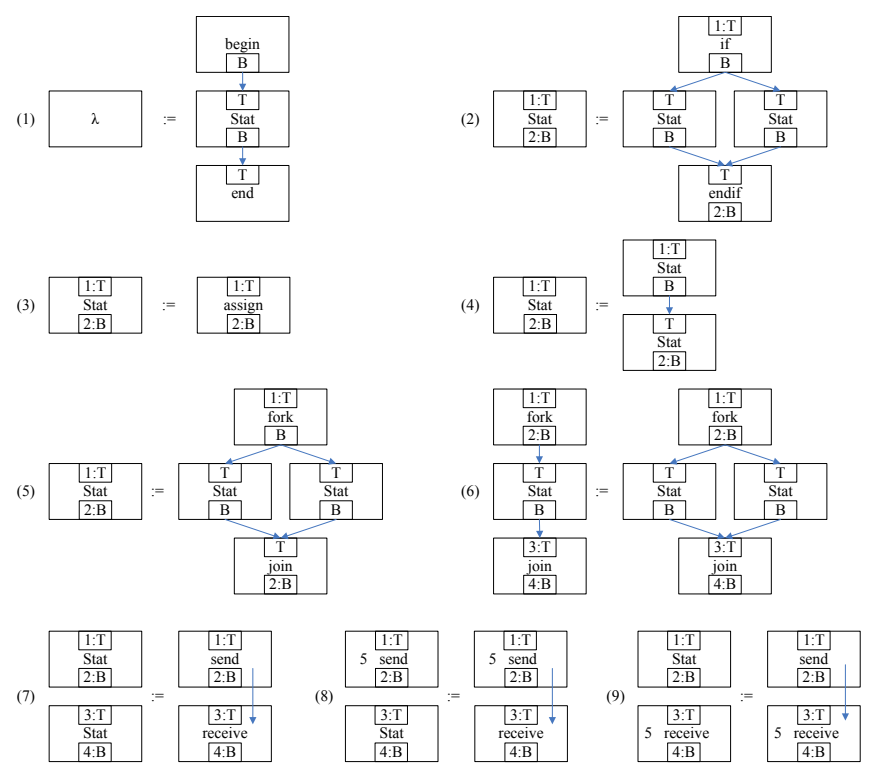

Figure 1. A graph grammar for process flow diagrams.

RGG is a context-sensitive graph grammar formalism [9]. It introduces a node-edge format to represent graphs in which each node is organized as a two-level structure, where the large surrounding rectangle is the first level, called super vertex, and other embedded small rectangles are the second level, called vertices. Either a vertex or a super vertex can be the connecting point of an edge. In addition to the two-level node structure, the RGG also introduces a marking technique that divides vertices into two categories: marked and unmarked ones. Each marked vertex of a production is identified by an integer that is unique in the left or right graph where the vertex lies. A production is properly marked if each marked vertex in the left graph has a counterpart marked by the same integer in the right graph, and vice versa. In the process of a production application, when a redex is matched in a host graph, each vertex that corresponds to a marked vertex in the left or right graph preserves its associated edges connected to nodes outside of the redex, which avoids the appearance of dangling edges during the subsequent subgraph replacement provided that an additional embedding rule is also enforced. The embedding rule states that if a vetex in the right (or left) graph of a production is unmarked and has an isomorphic vertex in the redex of a host graph, then all the edges connected to the vertex should be completely inside the redex. 
As an example, an RGG specifying process flow diagrams, which is slightly adapted from [8], is depicted in Figure 1.

\section{B. Partial and Total Precedence}

In this subsection, we take the RGG as the representative of implicit context-sensitive graph grammar formalisms to present partial and total precedence relations between graph productions. For the sake of clarity and simplicity, some basic concepts and notations are listed below. Note that graphs are directed ones in the node-edge format and only vertices in productions might be marked.

$R G G$ : A reserved graph grammar is a triple $(A, P, \Omega)$, where $A$ is an initial graph, $P$ a set of graph grammar productions, $\Omega$ a finite label set consisting of two disjoint sets $\Omega^{T}$ and $\Omega^{N T}$ (called terminal label set and nonterminal label set, respectively). For any production $p:=(L, R) \in P$, three conditions are satisfied: $R$ is non-empty, $L$ and $R$ are both over $\Omega$, and the size of $R$ are not less than that of $L$.

$p:=(L, R):$ A production with a pair of marked graphs: the left graph $L$ and right graph $R$. The notations $p . L$ and $p . R$ represent the left and right graph of a production $p$, respectively. For any graph $G, G . N$ and $G . E$ denote the set of nodes and edges, respectively; $n . V$ and $n . v$ denote the set of vertices and some vertex $v$ of a node $n$, respectively; and $G . V=\bigcup_{n \in G . N} n . V$ is the union of the sets of vertices of nodes in $G$; for any edge $e$, $s(e)$ and $t(e)$ represent the source and target vertex of $e$, respectively, and $l(e)$ is the label on $e . P_{L}=\{p . L \mid p \in P\}$ and $P_{R}=\{p . R \mid p \in P\}$.

$G_{1} \approx G_{2}: G_{1}$ is isomorphic to $G_{2}$.

Redex: A subgraph $X \subseteq H$ is a redex of graph $G$, denoted as $X \in R d(H, G)$, if $X \approx G$ under an isomorphic mapping $f$ and any vertex in $X$ that is isomorphic to an unmarked vertex in $G$ keeps its edges completely inside $X$.

$R d(H, G)$ : A set of redexes of marked graph $G$, which are subgraphs of graph $H$.

Merger: A graph $G$ is a merger of $G_{1}$ and $G_{2}$, if both $G_{1}$ and $G_{2}$ are subgraphs of $G$ and each node or edge in $G$ is either from $G_{1}$ or from $G_{2} \cdot \operatorname{Mrg}\left(G_{1}, G_{2}\right)$ is the set of mergers of $G_{1}$ and $G_{2}$.

Mcc: A mapping from graphs to the sets of maximal connected components contained in these graphs. A maximal connected component in a graph is a connected component being maximal. $\operatorname{Mcc}\left(P_{L}\right)=\{C \mid C \in \operatorname{Mcc}(p . L) \wedge p \in P\}, \operatorname{Mcc}\left(P_{R}\right)=$ $\{C \mid C \in \operatorname{Mcc}(p . R) \wedge p \in P\}$.

The following definitions excerpted from [21] are necessary to understand the notion of context and the approach to context computation.

Definition 1. Let $g g:=(A, P, \Omega)$ be an $R G G, p_{1}, p_{2} \in P$ be two productions, $C_{1} \in \operatorname{Mcc}\left(p_{1} . L\right)$ and $C_{2} \in \operatorname{Mcc}\left(p_{2} . R\right)$. If $\exists X \subseteq C_{2}$ such that $X \in \operatorname{Rd}\left(C_{2}, C_{1}\right)$, then $C_{1}$ is matched with $X$ in $C_{2}$, denoted as $C_{1} \approx X \subseteq C_{2}$; or concisely $C_{1}$ is included in $C_{2}$, denoted by $C_{1} \sqsubseteq C_{2}$.

This definition introduces the notion of inclusion between the components of productions, or to be exact, to locate a redex of a component of the left graph of one production in some component of the right graph of another production.

Let $U$ be some set and $S=(B, m)$ a multiset, where $B$ is the underlying set of elements and $m: B \rightarrow \mathbb{N}$ is a mapping from $B$ to the set $\mathbb{N}$ of positive natural numbers. $S \subseteq{ }^{\mathbb{N}} U$ if and only if $B \subseteq U$. In order to unambiguously reference to an element from a multiset, we stipulate that any two elements in a multiset $S$ have distinct identities even if they are the same element from the point of view of the underlying set $B$, and the identities of elements are not explicitly represented in context for the sake of conciseness.

Definition 2. Let $g g:=(A, P, \Omega)$ be an $R G G$, and $P_{L}$ and $P_{R}$ the sets of left and right graphs of productions in $P$, respectively. A set $S_{1} \subseteq \operatorname{Mcc}\left(P_{L}\right)$ is included in another multiset $S_{2} \subseteq \mathbb{N} \operatorname{Mcc}\left(P_{R}\right)$, denoted as $S_{1} \sqsubseteq S_{2}$, if there is a mapping $f: S_{1} \rightarrow S_{2}$ such that:

$$
\begin{gathered}
\cdot \forall C \in S_{1}(\exists X \subseteq f(C)(X \in R d(f(C), C))) \text {, and } \\
\cdot \forall C, C^{\prime} \in S_{1}\left(C \neq C^{\prime} \wedge f(C)=f\left(C^{\prime}\right) \rightarrow \exists X, X^{\prime} \subseteq\right. \\
\left.f(C)\left(X \in R d(f(C), C) \wedge X^{\prime} \in R d\left(f(C), C^{\prime}\right) \wedge X \cap X^{\prime}=\phi\right)\right) .
\end{gathered}
$$

The first condition states that for each component in $S_{1}$, there is an image in $S_{2}$ under the mapping f that contains a redex of it; and the second expresses that if two different components in $S_{1}$ have the same image in $S_{2}$, then the two corresponding redexes in it cannot overlap, which adheres to the redex definition in the RGG formalism.

Definition 3. Let $g g:=(A, P, \Omega)$ be an $R G G$, and $p_{1}, p_{2} \in$ $P$ be two productions, $p_{1}$ directly partially precedes $p_{2}$, denoted as $p_{1} \preccurlyeq_{d} p_{2}$, if $\exists S \subseteq M c c\left(p_{2} . L\right)$ such that $S \subseteq \operatorname{Mcc}\left(p_{1} . R\right)$. The direct partial precedence relation between them is denoted by the pair $\left\langle p_{1}, p_{2}\right\rangle$. The direct partial precedence relation on the set $P$ of productions is defined as $\preccurlyeq_{P}=\left\{\left\langle p_{1}, p_{2}\right\rangle \mid p_{1}, p_{2} \in\right.$ $\left.P \wedge p_{1} \preccurlyeq_{d} p_{2}\right\}$. The partial precedence relation between them is denoted by the pair $\left\langle p_{1}, p_{2}\right\rangle$.

The partial precedence relation is the closure of the direct partial precedence relation on a set $P$. Partial precedence is a kind of relation between a pair of components chosen from two distinct productions, whereas total precedence describes the same relation between two sets of components from a subset of productions and a single production respectively.

Definition 4. Let $g g:=(A, P, \Omega)$ be an $R G G, p \in P$, and $a$ multiset $P^{\prime} \subseteq \mathbb{N} P$. $P^{\prime}$ directly totally precedes $p$, denoted as $P^{\prime} \prec_{d} p$, if there is a surjective mapping $f: \operatorname{Mcc}(p . L) \rightarrow S t$ such that:

- $S t \subseteq \operatorname{Mcc}\left(P_{R}^{\prime}\right)$;

- $\operatorname{Mcc}(p . L) \sqsubseteq \operatorname{Mcc}\left(P_{R}^{\prime}\right)$ with respect to $f$;

- $\forall p^{\prime} \in P^{\prime}\left(\exists C \in \operatorname{Mcc}(p . L)\left(f(C) \in \operatorname{Mcc}\left(p^{\prime} . R\right)\right)\right)$.

The corresponding direct total precedence relation is denoted by the pair $\left\langle P^{\prime}, p\right\rangle$, and $p$ and $P^{\prime}$ are called the target production and preceding set, respectively. The direct total precedence relations on the set $P$ of productions is defined as $\prec_{P}=$ $\left\{\left\langle P^{\prime}, p\right\rangle \mid P^{\prime} \subseteq^{\mathbb{N}} P \wedge p \in P \wedge P^{\prime} \prec_{d} p\right\}$.

A direct total precedence relation specifies that a certain graph composed of the right graphs of a subset of productions 
contains a redex of the left graph of another production. Note that the third constraint on $f$ emphasizes that every production in $P^{\prime}$ takes part in $f$ with at least one of its components in the right graph. If a subset $P^{\prime}$ of $P$ forms such a relation with a production $p$, then it means that all the right graphs of $P^{\prime}$ must exactly comprise a redex of the left graph of $p$ with each one containing at least one redex of its components.

A total precedence relation $\langle M, p\rangle$ is composed of a set of direct total precedence relations and a set of linking relations on it. A compound precedence set consists of three parts: a multiset $T$ of productions from set $P$, a multiset $E$ of direct total precedence relations, and a set $R$ of linking relations on $E$. A compound precedence set $M$, together with a production $p$, forms a total precedence relation, on condition that a direct total precedence relation is established between the first part of $M$ and the production $p$.

\section{Definition of Context}

The sets of partial or total precedence relations with respect to a graph grammar establish an order of production applications, which can be exploited to discover potential situations in which any of the productions is applicable for derivation. We refer to these situations as contexts. Given two productions $p_{1}$ and $p_{2}$, if $p_{1}$ directly partially precedes $p_{2}$, then $p_{1}$. $R$ contains a context of $p_{2}$ or merely a portion of a context, depending on whether $p_{2} . L$ consists of only one or at least two maximal connected components. As for the former case, $\left\{p_{1}\right\} \prec_{d} p_{2}$ readily holds and a context of $p_{2}$ immediately follows; whereas in the latter case, a subset of productions involving $p_{1}$ that directly totally precedes $p_{2}$ is pursued so as to form a complete context for $p_{2}$. As a third case, a total precedence relation can be sought to build a rather deeper complete context.

Complete contexts of a production can be stratified in terms of the levels of corresponding total precedence relations from which they are generated. Roughly, a complete context that is built in the light of a precedence relation that corresponds to a rooted tree of depth $i$ is called a level- $i$ context, $i \geq 1$, and it degrades to a level-1 context when the relation is a direct one.

A complete context can be employed to extend the respective production to which it pertains. This is done by augmenting the context simultaneously to its both graphs and properly linking the two parts together respectively. A production $p$ equipped with a level- $i$ context is called a context- $i p$.

Definition 5. Let $g g:=(A, P, \Omega)$ be an $R G G, p \in P$, $\operatorname{Mcc}(p . L)=\left\{C_{1}, \cdots, C_{n}\right\}$, and $P^{\prime} \subseteq^{\mathbb{N}} P$. If $P^{\prime} \prec_{d} p$ with respect to some surjective mapping $f: \operatorname{Mcc}(p . L) \rightarrow S t=\left\{D_{1}, \cdots, D_{m}\right\}$ and $a$ set of redexes $X=\left\{X_{i} \mid X_{i} \in R d\left(f\left(C_{i}\right), C_{i}\right), 1 \leq i \leq n\right\}$, then the pair $(U, Z)$ is a level-1 context of $p$ with respect to $P^{\prime}$, $f$, and $X$, denoted as $c t_{p}\left(P^{\prime}, f, X\right)$, where $U=\bigcup_{1 \leq j \leq m} D_{j}^{\prime}$, $D_{j}^{\prime}=D_{j} \backslash \cup_{k_{j} \in K_{j}} X_{k_{j}} \quad, \quad K_{j}=\left\{l \mid f\left(C_{l}\right)=D_{j} \wedge 1 \leq l \leq n\right\}$, $Z=\cup_{1 \leq j \leq m} Z_{j}$, and $Z_{j}=\left\{e \in D_{j} . E \mid\left(s(e) \in X_{k_{j}} . V \wedge t(e) \in\right.\right.$ $\left.\left.D_{j}^{\prime} . V\right) \vee\left(s(e) \in D_{j}^{\prime} . V \wedge t(e) \in X_{k_{j}} . V\right) \wedge k_{j} \in K_{j}\right\}$. The sets $U$ and $Z$ are called the contextual graph and contextual connection, respectively.

Each component $D_{j}^{\prime}$ of the contextual graph is the rest graph of $D_{j}$, a component of the right graph of some production that contains one or more redexes $X_{k_{j}}$ of the components $C_{k_{j}}$, minus $X_{k_{j}}$, and each $Z_{j}$ is the collection of edges in $D_{j}$ that connect $D_{j}^{\prime}$ to all the redexes $X_{k_{j}}$.

Similar to Definition 5, the notion of level- $i$ context can be recursively defined.

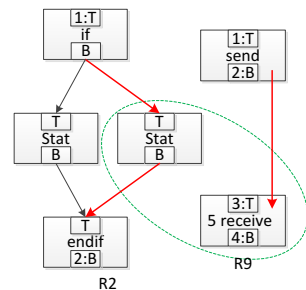

(a)
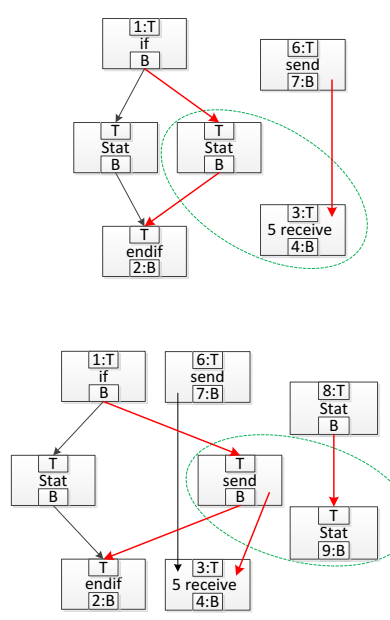

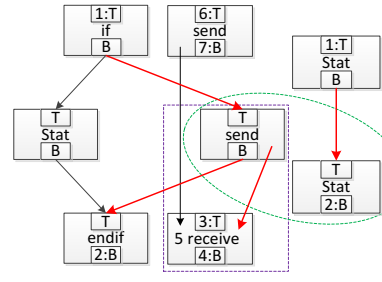

(b)
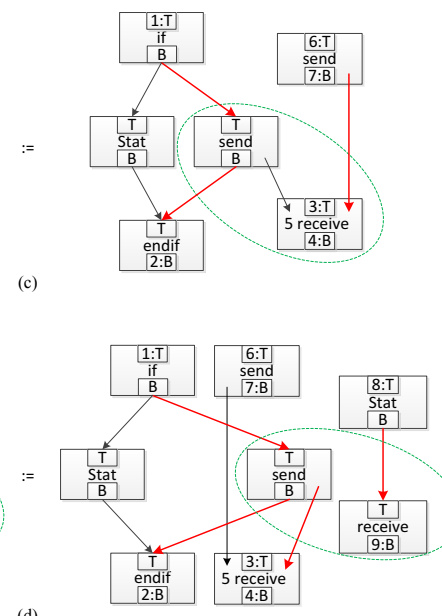

(d)
Figure 2. The contexts of a production and their extended productions. (a) A level-1 context of $p 9$. (b) A level-2 context of $p 8$. (c) A level-1 context equipped $p 9$. (d) A level-2 context equipped $p 8$.

Example 1. Two contexts of a production and their extended productions.

Two contexts at distinct levels of a production and their respective extended productions are demonstrated in Figure 2. A level-1 context of $p 9$ originated from the direct total precedence relation $\{p 2, p 9\} \prec_{d} p 9$ is shown in Figure 2(a), where the left component of the graph is $R 2$ (the right graph of production 2 ), the right one is $R 9$, the subgraph enclosed by the green dashed ellipse is the redex of $L 9$ (the left graph of $p 9$ ), and the context consists of two parts $U$ and $Z: U$ is the rest of the whole graph minus the redex and $Z$ the set of thick red edges that connect $U$ to the redex. The respective context- $1 p 9$, numbered as $p 10$, is depicted in (c), in which the two subgraphs surrounded by dashed green eclipses are the isomorphic image of the underlying production of $p 10$.

Figure 2(b) shows a level-2 context of $p 8$, which is created on the basis of the direct total precedence relation $U d(\{p 4, p 10\}) \prec_{d} p 8$, or equivalently, the total precedence relation $U l(\{p 4, p 10\}) \prec p 8$. In this graph, the left and right component is $R 10$ and $R 4$, respectively, the subgraph enclosed by the purple dashed rectangle is the isomorphic image of $U d(p 10)$, and the one surrounded by the green dashed eclipse is 
the redex of the left graph of $p 8$. Similarly, the corresponding context-2 $p 8$, numbered as $p 11$, is illustrated in (d).

\section{CONTEXT COMPUTATION}

In this section, an approach is presented for the computation of contexts in the RGG formalism based on the theoretical foundation reviewed in the preceding section.

The approach consists of four partially ordered algorithms, of which the first three deal with the computation of the set of direct partial precedence relations, the set of direct total precedence relations, and the set of total precedence relations with respect to a set of productions, respectively, and the last handles the computation of the contexts of a single production as well as the corresponding extended productions.

\section{A. Computation of Partial Precedence}

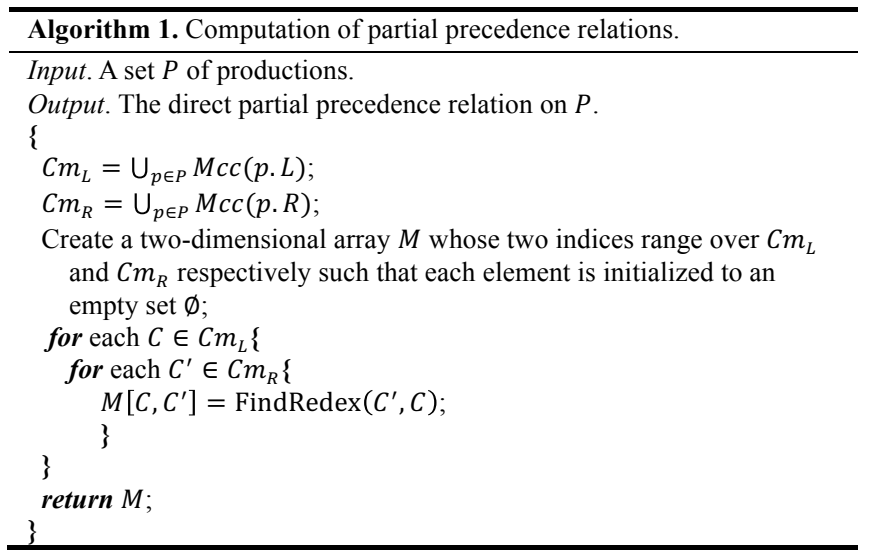

The first algorithm generates the partial direct precedence relation on a given set of productions. It consists of collecting all the components of the left and right graphs of the productions to create one set and another respectively, and then finding all the redexes of each component of the former in any one of the latter that is taken as the host graph. The output is a matrix such that each entry is assigned to a set of redexes (maybe empty) with respect to a pair of components from the two distinct sets that uniquely locate the entry in it.

\section{B. Computation of Direct Total Precedence}

The second algorithm figures out the direct total precedence relation on a set $P$ of productions, on the basis of the output of the first algorithm. First, it creates three one-dimensional arrays Llt, Rlt, Tpd and Fun which share a same index that ranges over $P$, to store the upcoming data. Then, it arranges the components of the left graph of each production $p$ into an ordered tuple form $\operatorname{Llt}[p]$ in a certain order, and for each element in this tuple, it generates a corresponding set that gathers all the redexes involved in the components from $\mathrm{Cm}_{R}$. Next, it conducts the Cartesian product Rlt $[p]$ of these sets of redexes in the same order as their counterparts in $L l t[p]$. As a result, every tuple in $R l t[p]$ is a redex of $L l t[p]$, since they are of the same length and each constituent of the former is a redex of the element of the latter to which it corresponds in terms of the tuple order. After that, by replacing each redex in a tuple of $R l t[p]$ with the underlying production whose right graph includes a component that contains this redex, it acquires a direct total precedence relation regarding $p$; this process repeats until all the relations regarding $p$, which comprise the set $D t p[p]$, are collected. Meanwhile, it establishes a mapping $h$ from $D t p[p]$ to a partition of $R l t[p]$ (i.e., a collection of disjoint nonempty subset of it that have it as their union) such that $h$ maps each tuple to the subset of $R l t[p]$, each of whose elements can be transformed to this tuple by the preceding replacement. Note that each element in $\operatorname{Dtp}[p]$ is a list of a multiset of productions in a predefined order. Nevertheless, a direct total precedence relation refers to a relation between a multiset of productions without any order and a production. To bridge this gap, it performs a partition of $D t p[p]$ in terms of such an equivalence relation that two lists are equivalent if both of them correspond to a same multiset, which produces $D p s[p]$ and $l$. In this way, it finally achieves the arrays Dps and Fun2 that can generate the direct total precedence relations regarding each production of $P$, together with the array Fun 1 that can produce the set of redexes with respect to any of these relations. For example, for each $S \in$ $\operatorname{Dps}[p],\langle S, p\rangle$ is a direct total precedence relation, and $\bigcup_{Q \in l(S)} h(Q)$ is the set of all the possible redexes.

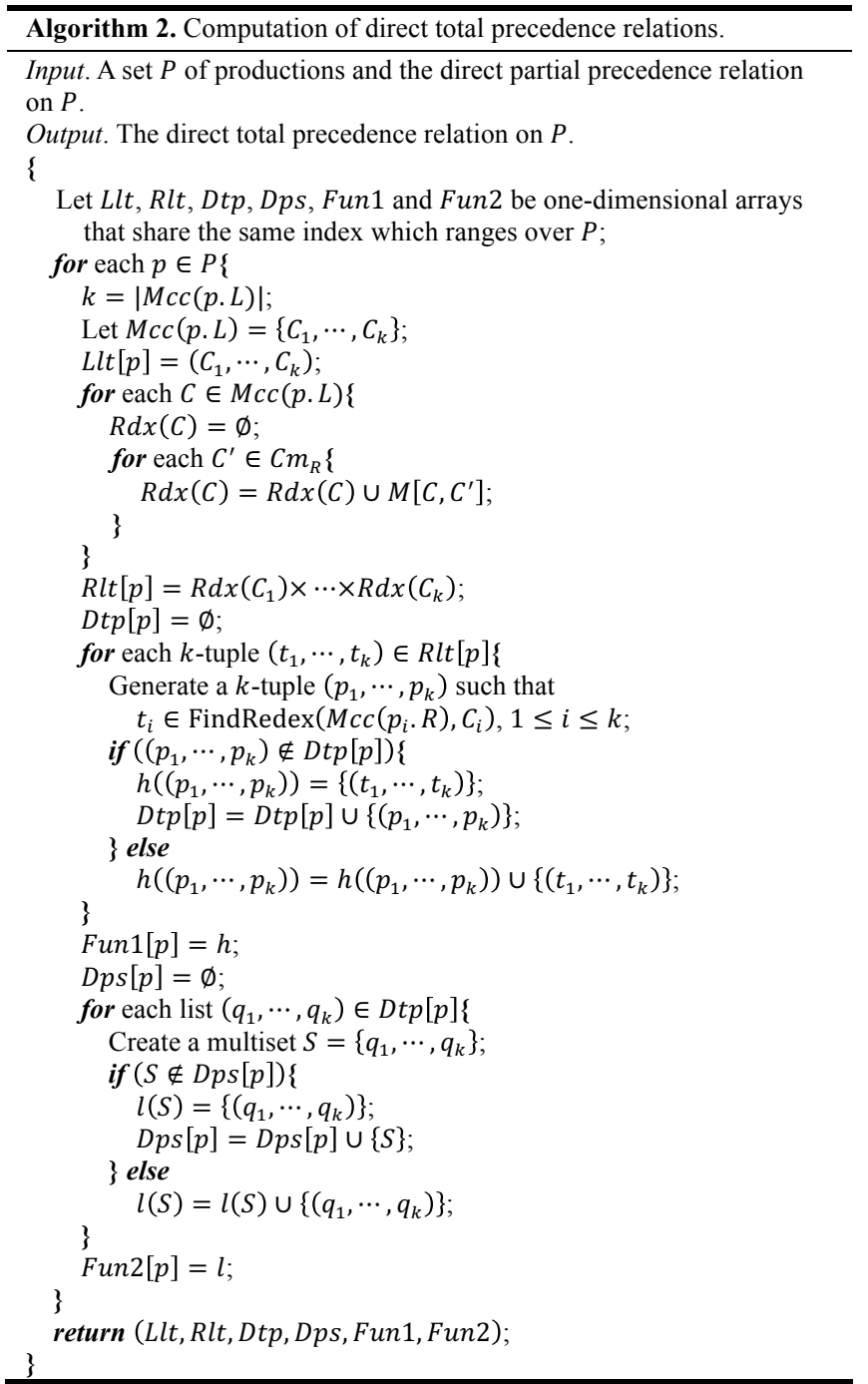

An underlying assumption for the algorithm is that any component in $C m_{L}$ or $C m_{R}$, as well as any redex of a component 
from $C m_{L}$ in another from $C m_{R}$, is uniquely identified. This is applicable from the perspective of algorithm implementation, as it can be achieved by assigning to each node of a production a unique number as its identity, and representing each redex as a triple $(S, f, i d)$ with $S$ the involved subgraph, i.e., the redex itself, $f$ the underlying mapping, and $i d$ the identifier of the right graph that contains the redex.

\section{Computation of Total Precedence}

The third algorithm describes the procedure of constructing all the total precedence relations of depth $k+1$ based on those of depth no more than $k$, with respect to a set $P$ of productions, where $k \geq 1$. Readily, the set of total precedence relations of any depth can be recursively generated from the fundamental set of direct total precedence relations by using it.



The algorithm consists of two tasks. First, it partitions the set Tpd into $|P|$ distinct subsets in terms of the root node of the rooted tree that each total precedence relation (i.e., a precedence structure) forms, which comprise the set Ptp.



Then, for any production $p$ in $P$, it constructs the set of all the total precedence relations of depth $k+1$ whose root elements share the same head $p$. More precisely, this set is the union of the subsets, each of which includes those relations whose root elements are a same direct total precedence relation with $p$ as the head. Thus, in terms of each of these relations, say $\left\langle P^{\prime \prime}, p\right\rangle$, the algorithm constructs a corresponding subset of all the relations of depth $k+1$ with $\left\langle P^{\prime \prime}, p\right\rangle$ as the root element. To this end, it first conducts the Cartesian product of $\left|P^{\prime \prime}\right|$ selected elements from Ptp whose indexes exactly comprises the multiset $P^{\prime \prime}$. Second, it screens the ordered tuples in the product to make sure that each chosen one can be utilized to generate a proper linking mapping from $P^{\prime \prime}$ to the union of $\{$ null $\}$ and the set of root elements of its constituents, and that the resulting precedence structure must be of depth $k+1$. The latter is guaranteed when one constituent of the tuple is of depth $k$. Third, for each chosen tuple, it generates the linking relation, which is composed of the head $\left\langle P^{\prime \prime}, p\right\rangle$, the set of tails, i.e., the root elements of the constituents of the tuple, and the newly created linking mapping, and then constructs the consequent precedence structure $(E, R)$, where $E$ and $R$ comprise all the involved direct 
total precedence relations and relevant linking relations, respectively.

\section{Computation of Context}

The fourth algorithm calculates all the level- $i$ contexts of a production and respective extended productions in terms of a total precedence relation, i.e., a precedence structure, with respect to it. It is known that a total precedence relation, i.e., a precedence structure $(E, R)$, forms a rooted tree in such a way that each direct total precedence relation in $E$ corresponds to a subtree of depth 1 and they are glued to each other in terms of the linking relations in $R$. The rooted tree structure offers a more easily comprehensible perspective of the algorithm.

The algorithm is composed of two consecutive tasks. The first task is to transform a total precedence relation (a rooted tree) into a set of direct total precedence relations (rooted trees of depth 1). To this end, the algorithm creates an empty set, adds the rooted tree to it, and repeats the subsequent four steps until all the elements in it become rooted trees of depth 1. First, it randomly selects a rooted tree from the set and locates an outmost subtree of length 1 (with the root node, say $p$ ); then, it figures out all the possible context- $i p$ 's according to the subtree, where $i \geq 1$; next, for each of those extended productions, say $p^{\prime}$, it constructs a new tree from the original one by pruning the subtree except the root (this node is preserved since it is also a leaf of another subtree to which this one is linked via a linking relation in $R$ ) from it and substituting $p^{\prime}$ for the root $p$, and puts it into the set; and finally, it deletes the originally selected tree from the set. After that, the set includes only rooted trees of depth 1 , any of which corresponds to a direct total precedence relation. In the second task, it constructs, for each element in the set, a level- $h$ context and an accompanying extended production as well.

\section{COMPLEXITY ANALYSIS}

In this section, the complexities of the proposed algorithms in the preceding section are analyzed.

Algorithm 1 calls a procedure $\operatorname{FindRedex}(H, G)$ to generate the set of subgraphs of $H$ that are redexes of the marked graph $G$. A procedure similar to the callee was proposed in [9] with time complexity $O\left(|H|^{|G|}\right)$, where $|H|$ or $|G|$ denotes the number of nodes involved in it. Then, the time complexity of the algorithm directly follows:

Proposition 1. The time complexity of Algorithm 1 is $O\left(m^{2} n^{2} r^{r}\right)$, where $m$ is the number of productions in $P, n$ is the maximal number of components in the left or right graphs of productions in $P$, and $r$ is the maximal number of nodes in any of the components.

Proposition 2. The time complexity of Algorithm 2 is $O\left((m n)^{n+1} r^{r n}\right)$, where $m, n$ and $r$ are as in Proposition 1.

Proof. The algorithm mainly consists of a for-loop that nests other three sequential for-loops. In the outmost loop, the first nested for-loop also nests another for-loop, which takes time $O\left(m n^{2}\right)$. The line next to it is the calculation of a $k$-ary Cartesian product over sets of redexes of components in $p . L$, each of which contains at most $m n \times r^{r}$ elements, where $m n$ and $r^{r}$ are the maximal number of elements in $C m_{R}$ and $M\left[C, C^{\prime}\right]$, respectively. Thus, the time complexity is $O\left(\left(m n r^{r}\right)^{n}\right)$, since $k$ equals to $n$ in the worst case. In the second nested for-loop, the first line takes $O(n)$, because the production to which each redex in the ordered tuple of Rlt corresponds is clearly indicated beforehand, according to the underlying assumption. So, this for-loop takes $O\left(n\left(m n r^{r}\right)^{n}\right)$. Moreover, the time complexity of the last nested for-loop is at most $O\left(n\left(m n r^{r}\right)^{n}\right)$, for the cardinality of $D t p[p]$ is no more than that of $R l t[p]$. In summary, the nested part of the outmost loop takes time $O\left(n\left(m n r^{r}\right)^{n}\right)$. Consequently, the time complexity of the whole procedure is $O\left((m n)^{n+1} r^{r n}\right)$, which is the product of the time complexity of the outmost for-loop and that of its nested part.

Proposition 3. The time complexity of Algorithm 3 is $O\left(m^{n+2} n l^{n}\right)$, where $m$ and $n$ are as in Proposition 1 , and $l$ is the cardinality of Tpd.

Proof. The algorithm consists of three sequential for-loops. It is evident that the first two loops take $O(n)$ and $O(l)$, respectively. The last one is a four-layer nested for-loops. According to the structure, its time complexity can be expressed as $O\left(d_{1} d_{2} d_{3} d_{4}\right)$, where $d_{1}, d_{2}, d_{3}$ and $d_{4}$ are the maximal number of times traversed in the outmost, second, third, and inmost for-loop, respectively. We proceed inwards from outside of the structure. First, $d_{1}$ is the number of productions in $P$, that is, $d_{1}=m$. Next, $d_{2}$ equals to the cardinality of $\preccurlyeq_{P}$, which is no more than $m \times m^{n}=m^{n+1}$. It is obvious that $d_{3}$ is actually the cardinality of the Cartesian product over $k$ sets from Ptp, where $k$ denotes the number of tails in a direct total precedence relation. Since Ptp is a partition of Tpd, each element of the former must be a subset of the latter. Thus, $d_{3}<l^{n}$. As for the inmost loop, $d_{4}$ readily equals to $n$, which is exactly the same as the exponent appearing in the preceding inequation. Consequently, the last structure takes $O\left(m^{n+2} n l^{n}\right)$, i.e., the product of the complexities of the four constituents. Readily, it is also the time complexity of the algorithm.

Theorem 1. The time complexity of Algorithm 4 is $O\left(\left(n ! r^{r n}\right)^{2 n^{h-1}}\right)$, where $n$ and $r$ are as in Proposition 1, and $h$ is the depth of the input total precedence relation.

Proof. The main part of the algorithm is a two-layer structure: a for-loop nested within a while-loop, followed by another forloop.

As to the former, the total number of times it is traversed is the product of that of the outmost while-loop and of the inmost for-loop. We consider the while-loop first. In the worst case, the input total precedence relation $\left(E_{0}, R_{0}\right)$ corresponds to a complete $n$-ary rooted tree of depth $h$. Then, the cardinality of $E_{0}$, i.e., the number of subtrees of depth 1 that compose it, can be expressed as:

$$
1+n+\cdots+n^{h-1}=\frac{n^{h}-1}{n-1}
$$

Suppose the number of times the inmost for-loop is traversed at the worst case be $w$. In each traversal of the while-loop, it takes a rooted tree out from $C p s$, and then puts as many as $w$ revised ones that comprise one less subtrees back into it. This process is repeated until each element in $C p s$ becomes a rooted tree of depth 1, i.e., it only involves a root element. Consequently, 
the maximal number of times the loop is traversed can be expressed as:

$$
1+w+w^{2} \cdots+w^{\left(\frac{n^{h}-1}{n-1}-1\right)}=\frac{w^{\frac{n^{h}-1}{n-1}}-1}{w-1}
$$

Further inference to Formula (2) can be done as follows:

$$
\begin{aligned}
& \frac{w^{\frac{n^{h}-1}{n-1}}-1}{w-1}<\frac{w}{w-1} \cdot w^{\frac{n^{h}-1}{n-1}-1}=\frac{w}{w-1} \cdot w^{\frac{n\left(n^{h-1}-1\right)}{n-1}} \\
& <\frac{w}{w-1} \cdot w^{2 n^{h-1}}=O\left(w^{2 n^{h-1}}\right)
\end{aligned}
$$

Note that $w$ exactly equals to the number of extended context-1 productions that can be produced from a direct total precedence relation, by Definitions 4.3 and 4.6. In the worst case, the relation consists of one head and $n$ tails, and each component of the latter's right graphs contains $r^{r}$ redexes of any component of the former's left graph. To be exact, of the loop variable $\left(t_{1}, \cdots, t_{k}\right)$ for the for-loop, each element $t_{i}$ can be any of the $r^{r}$ redexes of the corresponding component of the left graph in any component of any tail's right graph, where $1 \leq i \leq k$, and $k=$ $n$. Thus, for each permutation of the tails, there are $\left(r^{r}\right)^{n}=r^{r n}$ possible ordered tuples, where $n$ is the maximal number of choices for selecting one component from each tail, and $r^{r}$ is the maximal number of redexes in each component. Furthermore, the number of permutations for the tails is $n$ !. Therefore, $w=$ $n ! r^{r n}$. Substituting the result for $w$ in Formula (2) yields $\left(n ! r^{r n}\right)^{2 n^{h-1}}$.

As to the latter, the number of times it is traversed is $n^{h-1} \times n=n^{h}$.

Consequently, the time complexity of the algorithm is $O\left(\left(n ! r^{r n}\right)^{2 n^{h-1}}\right)$.

\section{DISCUSSION}

From the perspective of time complexity, the above four algorithms seem rather complicated at first glance. Nevertheless, they are applicable in practical scenarios due to the following three causes.

First, the parameters that characterize a graph grammar are usually small constants, and cannot change in any computation. That is, the parameters are the nature of a graph grammar that will not vary with host graphs in parsing or derivation processes under different situations.

Second, the worst cases theoretically assumed in the analysis of the complexities can rarely happen in practice, and they are frequently quite small number in practical applications. Notice that the number of redexes with respect to a direct total precedence relation is surprisingly $n ! r^{r n}$. However, this amount is merely a theoretical upper bound that accounts for all the possibly matched subgraphs, no matter in which situation all the redexes can simultaneously occur. An extreme situation in this case is a host graph where all the nodes in the host graph are labeled with the same symbol and the directed edges between them are completely connected. However, such host graphs can rarely be encountered in practice. As an example, consider the graph grammar depicted in Figure 1, the number of redexes with respect to a direct total precedence relation is theoretically
$2 ! 4^{2 \times 4}$, whereas in the practical computation it is less than 10 in most cases.

Third, the contexts of a graph grammar can be achieved as the output from merely one execution of the algorithms, and then they can be repeatedly utilized in the process of derivation and parsing of this grammar at any time afterwards.

Noticeably, the computation complexities for context can be directly applied to the practical graph grammars specifying realworld visual languages, e.g., BPMN (Business Process Model and Notation), ER diagrams, UML diagrams, WebML (Web Modeling Language), chemical diagrams, and so on, since these graph grammars are concrete examples of the underlying formalisms where the specification of nodes and edges in productions is entirely inherited from the formalisms.

\section{CONCLUSION}

Taking RGG as a representative formalism, this paper has proposed an approach to the computation of context on the basis of the formal definition of context, and presented the time complexities of the involved four algorithms. The approach can facilitate the applicability of implicit graph grammars, as contexts of the productions are essential information for the comprehension of graph grammars and efficiency improvement of parsing algorithms. Besides, the approach can be generalized to other implicit context-sensitive graph grammars without much effort.

In future work, further investigation will be made to explore more application scenarios of context, and a support system for context computation and visualization will be developed as well.

\section{ACKNOWLEDGMENT}

This work is supported in part by the National Science Foundation of China under grants 61170089 and 91318301.

\section{REFERENCES}

[1] S. K. Chang, "Visual languages: a tutorial and survey," IEEE Software, 4(1), pp. 29-39, 1987.

[2] G. Costagliola, V. Deufemia, and G. Polese, "Visual language implementation through standard compiler-compiler techniques," Journal of Visual Languages and Computing, 18(2), pp. 165-226, 2007.

[3] K. Marriott, "Constraint multiset grammars," IEEE Symposium on Visual Languages, St. Louis, Missouri, pp. 118-125, 1994.

[4] F.Ferrucci, G. Pacini, G. Satta, et al., "Symbol-relation grammars: a formalism for graphical languages," Information and Computation, 131(1), pp. 1-46, 1996.

[5] G. Rozenberg (Ed.), Handbook on Graph Grammars and Computing by Graph Transformation, vol.1: Foundations, World Scientific, 1997.

[6] G. Engels, H.J. Kreowski, G. Rozenberg (Eds.): Handbook of Graph Grammars and Computing by Graph Transformation, vol 2: Applications, Languages, and Tools, World Scientific, 1999.

[7] H. Ehrig, H. J. Kreowski, U. Montanari, and G. Rozenberg (Eds.), Handbook of Graph Grammars and Computing by Graph Transformation, vol.3: Concurrency, Parallelism, and Distribution, World Scientific, 1999.

[8] J. Rekers and A. Schürr, "Defining and parsing visual languages with layered graph grammars," Journal of Visual Languages and Computing, 8(1), pp. 27-55, 1997.

[9] D. Zhang, K. Zhang, and J. Cao, "A context-sensitive graph grammar formalism for the specification of visual languages," The Computer Journal, 44(3), pp.187-200, 2001.

[10] M. Nagl, "A tutorial and bibliographical survey on graph grammars," International Workshop on Graph Grammars and Their Application to 
Computer Science and Biology, Lecture Notes in Computer Science, vol. 73, Springer Verlag, 1979, pp. 70-126.

[11] M. Nagl, "Set theoretic approaches to graph grammars," International Workshop on Graph Grammars and Their Application to Computer Science, Lecture Notes in Computer Science, vol. 291, Springer Verlag, 1987, pp. 41-54.

[12] J. Kong, K. Zhang, and X. Zeng, "Spatial graph grammars for graphical user interfaces," ACM Transactions on Computer-Human Interaction, 13(2), pp. 268-307, 2006.

[13] C. Zhao, J. Kong, and K. Zhang, Program behavior discovery and verification: a graph grammar approach, IEEE Transactions on Software Engineering, 36(3), pp. 431-448, 2010

[14] Y. Liu, X. Zeng, Y. Zou, and K. Zhang, "A graph grammar-based approach for graph layout," Software: Practice and Experience, 49(8), pp. $1523-1535,2018$

[15] Z. Shi, X. Zeng, Y. Zou, et al., A temporal graph grammar formalism. Journal of Visual Languages and Computing, vol. 47, pp. 62-76, 2018.
[16] J. L. Pfaltz, A. Rosefeld, "Web grammars," International Joint Conference on Artificial Intelligence, pp. 609-619, 1969.

[17] X. Zeng, X. Han, and Y. Zou, "An edge-based context-sensitive graph grammar formalism," Journal of Software, 19(8), pp. 1893-1901, 2008. (in Chinese)

[18] Y. Liu, Z. Shi, and Y. Wang, “An edge-based graph grammar formalism and its support system," International DMS Conference on Visualization and Visual Languages, pp.101-108, 2018.

[19] Y. Zou, X. Zeng, and X. Han, "Context-attributed graph grammar framework for specifying visual languages," Journal of Southeast University (English Edition), 24(4), pp. 455-461, 2008.

[20] P. Bottoni, G. Taentzer, and A. Schürr, "Efficient parsing of visual languages based on critical pair analysis and contextual layered graph transformation," IEEE Symposium on Visual Languages, pp. 59-60, 2000

[21] Y. Zou, J. Lü, and X. Tao, "Research on context of implicit contextsensitive graph grammars," Journal of Computer Languages, in press, https://doi.org/10.1016/j.cola.2019.01.002 